\title{
THE FIFTIETH ANNUAL MEETING OF THE AMERICAN ASSOCIATION FOR THE ADVANCEMENT OF SCIENCE.
}

The fiftieth annual meeting of the American Association for the Advancement of Science at Denver August 24-31, 1901, was the first meeting of this association held west of the Missouri River. There were 306 members and associates in attendance and about 220 papers were read before the various sections. Inaugurating a policy already announced, the Council voted that "each affiliated society is entitled to elect one member who is a fellow of the Association, as its representative in the Council; and if the society contains more than twenty five members who are fellows of the Association it is entitled to two representatives in the Council." The American Mathematical Society would therefore be entitled to two representatives in the Council at its meetings with the Association, more than seventy of its members being also fellows of the Association.

The next meeting of the Association will be held at Pittsburg, June 28 to July 3, 1902, under the presidency of Professor Asaph Hall, of Harvard. Professor G. W. Hough, of Northwestern University, will be vice-president of Section A, and Professor E. S. Crawley, of the University of Pennsylvania, will be secretary. It was recommended that the next following meeting of the Association be held in Washington during the week including January 1, 1903.

The average attendance at the meetings of the section of mathematics and astronomy was about twenty-five. Most of the papers were followed by brief discussions. The officers of this section were: vice-president, James McMahon; secretary, G. A. Miller ; councilor, G. B. Halsted ; sectional committee, James McMahon, G. A. Miller, H. A. Howe, Florian Cajori, F. H. Loud ; member of the general committee, C. A. Waldo. The Council elected the following mathematicians and astronomers to fellowship in the Association :

S. I. Bailey, E. M. Blake, C. L. Bouton, S. J. Brown, Wm. Cain, Florian Cajori, W. W. Campbell, C. W. Comstock, S. J. Cunningham, H. S. Davis, E. A. Engler, Irving Fisher, T. S. Fiske, G. B. Germann, S. Hart, H. A. Howe, C. J. Keyser, C. J. Ling, E. Miller, F. R. Moulton, H. B. Newson, G. A. Osborne, C. A. Post, T. J. J. See, W. B. Smith, J. M. Taylor, H. W. Tyler, J. H. Van Amringe. 
On account of a change in the policy of the Association, the address of the vice-president of Section A will be delivered at the Pittsburg meeting. The following papers were presented at the meetings of this section :

(1) Professor G. B. Halsted : "Supplementary report on non-euclidean geometry."

(2) Professor H. A. Howe : "Kepler's problem for high planetary eccentricities."

(3) Professor H. A. Howe and Miss L. L. Stingley : "The great fire ball of December 7, 1900."

(4) Professor Flonian CAJORI : "Conditionally convergent series whose product is absolutely convergent."

(5) Professor Florian CaJori : "On the application of the fundamental laws of algebra to infinite series."

(6) Professor L. E. Dickson : "'On systems of isothermal curves."

(7) Mr. C. J. Keyser : "The plane geometry of the point in space of four dimensions."

(8) Professor H. A. Howe and Miss M. C. TAylor : "The next opposition of Eros."

(9) Professor T. J. J. SEe : "On the probable densities of the satellites of the solar system."

(10) Mr. H. M. PARkeurst : "Photometric observations of Eros."

(11) Dr. G. A. Mrluer : "On the history of several fundamental theorems in the theory of groups of finite order."

(12) Professor ARNold EMch : "On certain methods in the geometry of position.",

(13) Professor F. L. Chase : "The parallex of 54 Piscium and of Weisse $17^{\mathrm{h}}, 322 . "$

(14) Professor F. L. Chase : "The distance of the new star in Perseus."

(15) Mr. A. C. Sмiтh : "Hyperbolic curves of the $n$th order."

(16) Dr. F. R. Moulton: "A uniform method of determining the elements of orbits of all eccentricities from three observations."

(17) Dr. J. I. Hutchinson : "On the modular functions associated with the irrationality

$$
s^{3}=z(z-1)(z-x)(z-y) . '
$$

(18) Professor F. H. Loud and Mr. L. R. Ingersoll : "Some future solar eclipses, in particular that of June 8, 1918, total at Denver.'"

(19) Professor Alexander Macfarlane : "Bibliography of quaternions and allied systems." 
(20) Professor R. S. Woodward : "A summary of the salient effects due to the secular cooling of the earth."

(21) Professor R. S. Woodward : "The energy of condensation of stellar bodies."

(22) Professor C. J. LING: "The Bruce micrometer."

(23) Professor Cleveland AbBe: "The physical basis of long range weather forecasts."

(24) Mr. Jesse Pawling, JR.: "The metric system in the United States."

(25) Mr. Jesse Pawling, JR.: "The new bureau of standards."

Three of the titles which appeared on the preliminary programme are not reproduced here on account of the fact that these papers did not reach the secretary in time to be presented at the meeting. In the absence of the authors the papers by Professor See and Dr. Moulton were presented by Professor Howe. The papers by Professors Dickson and Macfarlane, Dr. Hutchinson, and Messrs. Keyser and Parkhurst were read by the secretary. In the absence of the member who was to present Professor Abbe's paper it was read by title. Abstracts of the papers, in order, are given below.

Professor Halsted's report was supplementary to the one read at the Columbus Meeting two years ago and printed in Science. It was mainly devoted to the advances made during the last two years. The advances contained in Dehn's article (published in the Mathematische Annalen, 1900) and in Hilbert's "Grundlagen der Geometrie," 1899, were especially emphasized. The report will appear in Science.

The solution of the equation $M=E-e \sin E$ is commonly called Kepler's problem. The known quantities $e$ and $M$ are respectively the eccentricity and the mean anomaly $E$ is to be found. The purpose of Professor Howe's first paper is to develop a direct method of solving Kepler's problem for planetary orbits of high eccentricity, which shall be more expeditious than any heretofore discovered, and shall be sufficiently accurate to meet the most exacting requirements of astronomers.

Professor Howe's second paper was devoted to the methods employed to collect data in reference to the great fire ball under consideration and to a discussion of its path. These two papers will appear in the Astronomical Journal and Popular Astronomy respectively. 
In an article on "Divergent and conditionally convergent series whose product is absolutely convergent," in the Transactions of the American Mathematical Society, volume 2, 1901, p. 25, Professor Cajori gave special cases in which an absolutely convergent series is obtained as a result of multiplying two conditionally convergent series together or one conditionally convergent series by a divergent series. But the sum of one of the two factor series of each pair given in that article is zero. In the present paper it is shown that this is not a necessary property of conditionally convergent series whose product is absolutely convergent, and that the $n$th sum of such series may be of the degree $-r$ with respect to $n$, where $\frac{1}{2}<r \equiv 1$.

In his second paper Professor Cajori considers the behavior of infinite series with respect to the laws of algebra under two heads: An inquiry into the validity of the laws (1) when applied to the terms of an infinite series ; (2) when applied to the infinite series themselves. The second inquiry, when made for the multiplication of series, leads to the conclusion that in this operation (assuming Cauchy's definition for the product of two infinite series), the associative, commutative and distributive laws are obeyed.

The two series obtained by removing the parentheses from the series

$$
\begin{aligned}
& S_{1} \equiv \sum_{p=0}^{p=\infty}\left(\frac{1}{4 p^{r}+1}-\frac{1}{4 p^{r}+4}+\frac{1}{4 p^{r}+1}-\frac{1}{4 p^{r}+4}\right) \\
& S_{2} \equiv \sum_{p=0}^{p=\infty}\left(\frac{1}{4 p^{r}+4}+\frac{1}{4 p^{r}+4}-\frac{1}{4 p^{r}+1}-\frac{1}{4 p^{r}+1}\right),
\end{aligned}
$$

where $\frac{1}{2}<r \equiv 1$, are conditionally convergent, but their product is absolutely convergent. Hence, $\left(S_{1} S_{2}\right)\left(S_{1} S_{2}\right)\left(S_{1} S_{2}^{\prime}\right)$ is absolutely convergent. But $\left(S_{1} S_{2}\right)\left(S_{1} S_{2}\right)\left(S_{1} S_{2}\right)=S_{1}^{3} \cdot S_{2}^{3}$, and $S_{1}^{3}$ and $S_{2}^{3}$ are each divergent when $r<\frac{2}{3}$. Hence, when $\frac{1}{2}<r<\frac{2}{3}, S_{1}^{3}$ and $S_{2}^{3}$ are two divergent series whose product is absolutely convergent.

The object of Professor Dickson's paper is to give an elementary geometrical definition of a system of sothermal curves in the plane. The definition is readily extended to families of curves on any algebraic surface. Two families of curves are discussed at length. From these the general definition is apparent. The paper has been published in the October number of the American Mathematical Monthly. 
Mr. Keyser investigated the point (in 4-space) regarded as the assemblage of all the lineoids $(i$. e., ordinary 3 -fold spaces), planes, and lines containing it. This space is 3-dimensional in lineoids and in lines, the lineoid and the line being reciprocal elements ; it is 4-dimensional in planes, the plane being self-reciprocal. The plane being taken as element, a theory results which is in its analytical aspect identical with the Plücker line theory of the lineoid, while the two theories are geometrically disparate. The subject was treated under the following six headings: Introductory considerations, concerning certain metric relationships, homogeneous coördinates of the plane, the linear complex of planes, linear congruences of planes, and projective transformations by means of complexes.

In his third paper, which will appear in Popular Astronomy, Professor Howe explained the computations which have been made for the rediscovery and observation of Eros when the conditions will become favorable. A chart giving the path of Eros through the sky was exhibited, and also a pasteboard model of the orbits of the earth and the planet, showing their positions at favorable oppositions.

Professor See points out the difficulty of measuring the angular diameters of very small bodies, on account of the tremors of the atmosphere, and then considers the densities of the great planets. He concludes that the average density of the four inner planets is 4.25 , that of the outer planets 1.50 , while that of the satellites is 2.36 .

Mr. Parkhurst gave the results of observations extending from September 13th to March 22d and comprising 382 double extinctions in comparison with a large number of standard stars, including four other asteriods. The constant of brightness was ascertained to be 9.78 magnitude and the constant factor for phase angle .037 .

Dr. Miller's historical account will appear in Science.

The paper by Professor Chase was supplementary to a paper read by him before this section a year ago under the title, "The series of parallaxes of large proper motion stars made with the Yale heliometer," a research begun in 1892, the observational part of which was finished the present year. In that paper the author had stated that the results of a preliminary solution indicated two of the 97 stars under 
investigation to possess a parallax of nearly $0^{\prime \prime} .25$, which values, if confirmed by further observation, would place them among the first ten or twelve nearest stars, so far as at present known. These two stars have been further investigated, two additional pairs of comparison stars being selected for each of them, and the observations with the original pairs repeated at the same time. Altogether there were 56 observations on 54 Piscium and 54 on Weisse $17^{\mathrm{h}}, 322$, distributed as follows :

\section{Piscium, Mag. 6.2.}

Series I. 12 obs. with a \& b Mags. $7.5 \& 7.3$ (orig.)

Series II. 12 obs. with a \& b

Series III. 16 obs. with c \& d Mags. $8.7 \& 8.7$

Series IV. 16 obs. with e \& f Mags. $7.5 \& 5.5$

Weisse 17, 322 Mag. 8.0.

Series I. 10 obs. with a \& b Mags. 7.0 \& 8.0 (orig.)

Series II. 12 obs. with a \& b

Series III. 16 obs. with c \& d Mags. $7.2 \& 5.5$

(rep.)

Series IV. 16 obs. with e \& f Mags. $8.6 \& 7.2$

The observations treated in the customary way and the equations derived therefrom being solved, the following results were obtained:

for 54 Piscium from

$$
\begin{array}{lll}
\text { Series I. } \pi=+0.241 \pm 0.026 & \text { Wt. } 36.10 \\
\text { Series II. } \pi=+0.081 \pm 0.017 & \text { Wt. } 42.26 \\
\text { Series III. } \pi=+0.183 \pm 0.035 & \text { Wt. } 59.53 \\
\text { Series IV. } \pi=+0.055 \pm 0.023 & \text { Wt. } 51.40
\end{array}
$$

for Weisse 17,322 ,

$$
\begin{array}{lrl}
\text { Series I. } \pi=+0.218 \pm 0.030 & \text { Wt. } 33.60 \\
\text { Series II. } \pi=+0.189 \pm 0.034 & \text { Wt. } 35.08 \\
\text { Series III. } \pi=+0.198 \pm 0.022 & \text { Wt. } 45.87 \\
\text { Series IV. } \pi=-0.047 \pm 0.031 & \text { Wt. } 38.92
\end{array}
$$

When the new star in Perseus first appeared last February Professor Chase undertook to determine, if possible, the parallax of this most remarkable object. So far as is known to the author, no one has as yet succeeded in determining the distance of one of these new stars. In 1892 he began a series of observations on Nova Aurigæ for the same purpose, but it will be remembered that this star rapidly diminished in brilliancy, though with several fluctuations, and 
was not observable with the Yale heliometer for more than two or three months, which would not give a very sensible parallax factor. With Nova Persei conditions have been much more favorable, and even now the star is conspicuously brighter than the brightest comparison star employed, which was, according to Argelander, of the 7.4 magnitude.

There was but a single pair of comparison stars suitable for the purpose, viz., B.D. $+43^{\circ}, 720$ Mag. 7.4 and B.D. $+43^{\circ}, 766$ Mag. 8.0. Calling the first $a$ and the second $b$, the position angles were respectively about $252^{\circ}$ and $94^{\circ}$, and the distances, $2900^{\prime \prime}$ and $2700^{\prime \prime}$ from the Nova.

The plan was to make the observations in the usual symmetrical order $N a, N b, N b, N a$, so as to eliminate, as far as possible, the effect of refraction and other effects which may vary with the time. Since the distance $a b$ was not beyond the range of the heliometer it was thought expedient to measure this distance a'so each night, and thus have besides the sums of the distances an independent basis for correcting for changes in the scale value from night to night. Each night's work, then, consists of six observations of distance, each of four pointings in reversed positions of the instrument, as follows: $N a, N b, a b, a b, N b, N a$. A number of calculations were based upon these observations. Should the star remain sufficiently bright for another six months, it will then be possible to determine the effect of proper motion and hence give a definitive result.

In a plane are given $n$ straight lines. Any point in the plane is taken as origin and through this a line is drawn, intersecting the $n$ lines in $n$ points. In this manner $n$ segments measured from the origin, are obtained on the line through the origin. The algebraic sum of these segments taken on this rame line will determine a point $P$ which will describe a curve of the $n$th order as the ray through the origin rotates through $360^{\circ}$. Mr. Smith considered the locus of $P$ and linear transformations of some of its special forms.

The methods of determining orbits which are in most general use were devised by Gauss one hundred years ago. They are different for orbits in which the eccentricities are less than, equal to, and greater than unity, although there is no singularity which is essential to the problem for the eccentricity equal to one. The method of Dr. Moulton's paper is uniform for all orbits, it is considerably more convenient than that of Gauss, and the radius of convergence of the series employed is examined in each case. 
The longitude of the node and the inclination are computed by the usual methods, which are satisfactory, and the heliocentric distances and the arguments of the latitude at the epochs of the three observations are computed as in the method of Gauss.

Let $u_{1}, u_{2}, u_{3}, r_{1}, r_{2}, r_{3}$ represent the arguments of the latitude and the heliocentric distances at the epochs of the three observations. Then the parameter $p$ is defined by the equation

$$
k \sqrt{p}\left(t_{3}-t_{1}\right)=\int_{u_{1}}^{u_{3}} r^{2} d u
$$

where $r^{2}$ is expressed as a series in $u$ whose radius of convergence is determined as a function of $u_{2}$ and $e$. It is shown how the coefficients are to be found. The eccentricity $e$ and the longitude $\omega$ of the perihelion from the node are given by

$$
\begin{aligned}
& e \sin \left(u_{1}-\omega\right)=\left\{\frac{p-r_{1}}{r_{1}} \cos \left(u_{3}-u_{1}\right)\right. \\
& \left.-\frac{p-r_{3}}{r_{3}}\right\} \operatorname{cosec}\left(u_{3}-u_{1}\right),
\end{aligned}
$$

$e \cos \left(u_{1}-\omega\right)=\frac{p-r_{1}}{r_{1}}$

The time of perihelion passage is determined from the law of areas.

The paper by Dr. Hutchinson aims to extend the results obtained by Picard in his memoir "Sur des fonctions de deux variables indépendantes analogues aux fonctions modulaires" (Acta Mathematica, volume 2, p. 114). Picard considers in the first place the integrals of the first kind and, in particular, the moduli of periodicity of the normal integrals. By changing the values of $x, y$ in a continuous manner so as to return finally to their initial values, the moduli undergo a linear transformation, which can be represented by a linear transformation on two parameters $u, v$ in terms of which all the moduli are rationally expressible. These transformations, forming an infinite group $G$, can be generated by five special ones $S_{1}, S_{2}, \ldots, S_{5}$, the explicit equations for which were given by Picard in a subsequent paper (Acta Mathematica, volume 5). The two variables $x$, $y$ are then automorphic functions of $u, v$, and all functions belonging to the group can be rationally expressed in terms of these. 
According to theorems previously obtained by Picard, there exist functions possessing a pseudo-automorphic character, exactly analogous to the fuchsian theta functions which Poincaré uses in connection with the automorphic functions of a single variable. These functions can be constructed out of the theta constants. In order to do this it is necessary to determine the effect of the transformations of the group $G$ on the Jatter, which is accomplished by means of the transformation theory of the theta functions. A table is constructed by means of which pseudo-automorphic functions can readily be constructed.

The tables used in computing the circumstances of the eclipses discussed by Professor Loud are those "On the recurrence of solar eclipses" published by Professor Simon Newcomb in 1879. After some remarks upon the limits within which the errors of such a computation may be expected to fall, the results of what seems the preferable combination of Professor Newcomb's tabulated data are stated as follows :

On June 8, 1918, the moon's shadow passer across the United States from northwest to southeast, covering Denver from $4^{\mathrm{h}} 22^{\mathrm{m}} 59^{\mathrm{s}}$ P. M. to $4^{\mathrm{h}} 24^{\mathrm{m}} 23^{\mathrm{s}}$-a period of $1^{\mathrm{m}} 24^{\mathrm{s}}$; while on the central line the duration is $1^{\mathrm{m}} 33^{\mathrm{s}}$. The width of the shadow path is about $59.3^{\mathrm{m}}$ and the velocity of the shadow $2,900^{\mathrm{m}}$ an hour. The eclipse will be visible from Chamberlin Observatory, Denver, and Mt. Arapahoe, Grand Co.

Sept. 10, 1923. An eclipse of duration $3^{\mathrm{m}} 24^{\mathrm{s}}$ is total from San Diego, Cal., eastward along a line near the United States and Mexican boundary. Width of shadow path 102 miles.

Jan. 24, 1925. An eclipse visible (as total) from northern Michigan to New Haven, Ct., reaching the latter point at $9^{\mathrm{h}} 5^{\mathrm{m}}$ A. M., and lasting $2^{\mathrm{m}} 8^{\mathrm{s}}$.

Two maps of the Denver eclipse were shown.

The association for the promotion of quaternions and allied mathematics has in preparation a bibliography of all the literature of the subject. The field embraces all that has been written on what is called geometric algebra, or space analysis, and its three main subdivisions are quaternions, Ausdehnungslehre, and geometric algebra previous to Hamilton and Grassmann. As regards the first branch, the papers of Hamilton himself are numerous, and he has been followed by about one hundred writers. The writings 
of Grassmann are also numerous, and he has been followed by about the same number of writers. It is desired to make the bibliography as complete as possible, and Professor Macfarlane requests mathematicians to coöperate by sending to him the necessary data about their own writings, or any rare writings in their possession.

The effects summarized in Professor Woodward's first paper are: (a) The slow process of heat conduction in the earth's crust, leading to the conclusion that nothing less than a million years is a suitable time unit for recording the historical succession of events. (b) The insignificant modifications of the process of conduction arising from the hydrosphere, leading to the conclusion that secular cooling goes on substantially as if the earth had neither atmosphere nor ocean. (c) The resultant effect on the lithosphere of secular contraction and the process of isostacy. (d) The effects of secular contraction on the length of the sidereal day.

The problem considered in Professor Woodward's second paper is that of the energy due to the gravitational condensation of gaseous matter from a state of infinite diffusion to a finite spherical mass in which Laplace's law of density holds. The problem is worked out in its generality, formulas specifying the distribution of density, pressure, and potential in the mass being given. Special attention is given to the probable case of the fixed stars of a vanishing surface density.

Mr. Ling exhibited and gave a description of the filar micrometer which the late Miss Caroline W. Bruce presented to Chamberlin Observatory.

The object of Professor Abbe's paper is to direct the attention of mathematical physicists toward meteorology and to set before the ordinary student of the daily weather map those fundamental mechanical and thermal principles that govern the phenomena of the atmosphere. The author believes that the present empirical rules and approximate general methods will eventually be replaced by precise methods of a mixed analytical, graphical, and numerical character, and that the time is now ripe for students of hydrodynamics and theoretical physics to apply themselves to the practical problems whose solution means so much to the progress of man in conquering nature. The author explained and stated many of the equations of motion and of condition and as to others he referred to special treatises. He indi- 
cated the general character of the laws of resistance of continents, mountains, and oceans to the motion of the atmosphere as also the probable general characteristics of the desired solutions of the whole system of equations. His fundamental departures from all previous analytical studies consisted in the recognition of a so-called convectional friction or resistance due to the interference with each other of ascending and descending currents of air, and in the recognition of the division of the globe into land and water hemispheres having their poles at London and anti-London respectively.

Mr. Pawling's first paper gave a brief history of the action of Congress in regard to weights and measures. 'The author also spoke of the "English decimal association" and the good that a similar association might do in this country. In his second paper he gave an account of the new National bureau of standards which is to be located in the suburbs of Washington. It has an appropriation of $\$ 300,000$ and a laboratory costing about $\$ 200,000$ is to be erected. The Bureau will employ a number of young men just graduated from universities giving them opportunities to develop along the lines which they wish to follow. It will also employ specialists to do work in their line. It standardizes three grades of weights and measures : $1^{\circ}$ Those for commercial use, $2^{\circ}$ those for manufacturing and technical processes and professions, $3^{\circ}$ those for extreme accuracy for scientific purposes. Professor Nichols, of Cornell, added that the prospects seemed very good that the United States was about to take a place among the leading nations in scientific work along this line.

STANFORD UNIVERSTTY.

G. A. Miller.

\section{RIEMANN-WEBER : PARTIAL DIFFERENTIAL EQUATIONS OF MATHEMATICAL PHYSICS.}

Die Partiellen Differentialgleichungen der Mathematischen Physik. Nach Riemann's Vorlesungen in vierter Auflage neu bearbeitet von Heinrich Weber, Professor der Mathematik an der Universität Strassburg. Braunschweig, Friedrich Vieweg und Sohn. Erster Band, 19(1)0, xvii + 506 pp. Zweiter Band, 1901, xi + 527 pp.

RiemanN's lectures on the partial differential equations of mathematical physics and their application to heat conduc- 